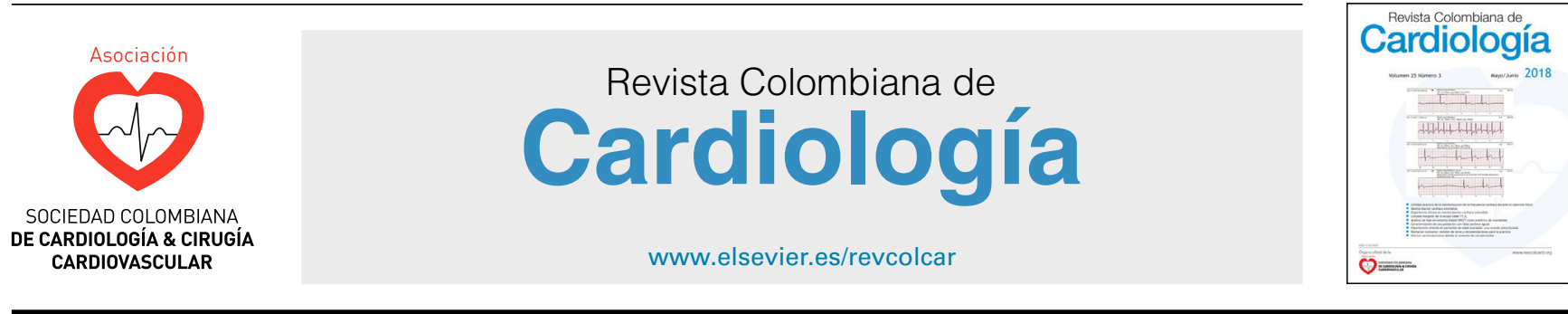

\title{
EDITORIAL
}

\section{Investigación e innovación para la salud: perspectivas y compromisos}

\section{Research and innovation for health: Perspectives and commitments}

\author{
Dora Inés Molina de Salazar ${ }^{\mathrm{a}, \mathrm{b}, \mathrm{c}, \mathrm{d}, *}$ y Carlos Eduardo Rivera-Molano ${ }^{\mathrm{c}, \mathrm{e}}$
}

\author{
a Facultad Ciencias para la Salud, Universidad de Caldas. Manizales, Colombia \\ b Comité Editorial de la Revista Colombiana de Cardiología. Bogotá, Colombia \\ c IPS Médicos Internistas de Caldas. Manizales, Colombia \\ d Asociación Colombiana de centro de investigación clínica - ACIC. Bogotá, Colombia \\ e Universidad de Manizales. Manizales, Colombia
}

Recibido el 25 de junio de 2019; aceptado el 27 de junio de 2019

La necesidad del reconocimiento y la publicación de todos los resultados obtenidos de procesos académicos y científicos se puede remontar al siglo $\mathrm{XVII}^{1}$ y ha sido una tendencia que ha cobrado importancia, a tal punto que en la actualidad dicha difusión se ha convertido en un indicador de desarrollo de una sociedad, que ha hecho que este tema sea marcado por ideas relacionadas con la productividad y la competitividad.

En particular, en la comunidad Latinoamericana ${ }^{1}$ el tema en mención se mide de acuerdo con el número de publicaciones que se hacen en revistas científicas, especialmente en aquellas calificadas y medidas, tanto en el ámbito nacional como en el internacional; además, se pone en consideración el nivel de colaboración con el cual se realizó la producción y el número de veces que es citado dicho artículo.

Este tipo de indicadores ha comenzado a utilizarse en conjunto con otros como el producto interno bruto y el índice de inversión, en investigación de una nación, para evaluar el impacto que puede llegar a tener la producción

\footnotetext{
* Autor para correspondencia.

Correo electrónico: doraines56@gmail.com (D.I. Molina de Salazar).
}

científica sobre el desarrollo económico del país. Lo anterior justifica la revisión de algunos tópicos relacionados con la medición de la producción científica en Colombia.

El primer dato a analizar está relacionado con la evaluación de Scimago Journal \& Country Rank, una plataforma que además de ofrecer una amplia gama de revistas científicas, muestra diferentes tipos de evaluación y clasificación de países, regiones del mundo y áreas del conocimiento en lo que atañe a la producción de documentos.

Para 2017, de acuerdo con la información de dicha plataforma ${ }^{2}$, Colombia ocupaba el cuarto puesto en América Latina en cuanto a producción de documentos publicados allí para el área de profesiones de la salud, con 122 documentos, después de Brasil, Chile y México, respectivamente y con un índice $\mathrm{H}$ de 36 . Para este mismo año, en relación con la publicación de documentos del área de Medicina, ocupó el quinto lugar con 2.957 artículos y obtuvo un índice $\mathrm{H}$ de 185, después de Brasil, México Argentina y Chile, respectivamente.

Se puede observar cómo la producción nacional en Medicina supera la producción clasificada como general en salud, marcando una ventaja de la misma frente a otras áreas similares. De igual forma, se observa el esfuerzo que se ha generado por parte de los profesionales y especialistas en 
Tabla 1 Revistas indexadas en clasificación de Publindex por áreas. 2017

\begin{tabular}{lll}
\hline Área & $\begin{array}{l}\text { Número de } \\
\text { revistas }\end{array}$ & $\begin{array}{l}\text { Porcentaje } \\
(\%)\end{array}$ \\
\hline Ciencias sociales & 112 & 45,53 \\
Humanidades & 39 & 15,85 \\
Ciencias médicas y de la & 33 & 13,41 \\
$\quad$ salud & & \\
Ingeniería y tecnología & 30 & 12,20 \\
Ciencias naturales & 18 & 7,32 \\
Ciencias agrícolas & 14 & 5,69 \\
Total & 246 & 100.00 \\
\hline
\end{tabular}

Medicina y las instituciones de formación superior en este sentido, mejorando así la visibilidad del país en el ámbito internacional.

Teniendo en consideración parámetros de medición locales, en la última clasificación de Publindex en $2017^{3}$, se aprecian 246 revistas clasificadas en A1, A2, B y C, distribuidas en seis áreas: Ciencias médicas y de la salud, Ciencias agrícolas, Ciencias sociales, Humanidades, Ciencias naturales, e Ingeniería y tecnología (tabla 1).

En la tabla se evidencia cómo en las clasificaciones nacionales se unifican tanto áreas de la salud como áreas médicas, mientras que en la clasificación Scimago Journal \& Country Rank están separadas; adicionalmente, se observa cómo las Ciencias médicas y de la salud ocupan el tercer lugar en número de revistas inscritas y calificadas por Publindex. En esta clasificación, de 33 revistas científicas indexadas en el área de Ciencias médicas y de la salud para el año 2017, ninguna clasifica como $A 1$, dos $(6,06 \%)$ están clasificadas en categoría $A 2$, once $(33,33 \%)$ en categoría $B$ y veinte en $(60,61 \%)$ categoría C, dentro de las cuales figura la Revista de la Sociedad Colombiana de Cardiología.

Se identifica así uno de los puntos que requiere más atención debido a que como área de conocimiento "Ciencias médicas y de la salud"', se encuentra 32,12 puntos porcentuales por debajo de "Ciencias sociales", y dada la cantidad de oportunidades que existen de generar conocimiento y estimular la apropiación del mismo, específicamente en Medicina, la oportunidad de fortalecer los grupos de investigación y las revistas científicas del área, es grande.

Este tipo de ejercicio es solo una forma de visualizar la producción científica, debido a que desde hace varios años han comenzado a desarrollarse evaluaciones bibliométricas encaminadas a analizar este tema desde una perspectiva investigativa. Un ejemplo de esto es el artículo "La producción científica colombiana en SCIELO: un análisis bibliométrico"' 4 en el cual se estima que el $61,5 \%$ de los artículos publicados están relacionados con instituciones universitarias. En este mismo documento ${ }^{4}$, se establece que de 2002 a 2013, la mayor producción de publicaciones ocurrió a partir de 2008 con el mayor pico en 2012; sin embargo, de estos documentos el $77,74 \%$ no han sido citados en otras publicaciones y gran número de estas producciones son de autoría únicamente colombiana, es decir, no cuentan con colaboración internacional.

En el periodo 2002 a 2013, las dos revistas que más publicaciones presentaron fueron Biomédica y Revista de
Salud Pública, teniendo en cuenta que este análisis incluyó todas las áreas disciplinares. Además, se observa que un $81,91 \%$ de la producción colombiana se publica en el ámbito nacional, cifra que indica la poca producción científica que se proyecta en el campo internacional.

Pese a que se pueden resaltar varios puntos en este artículo, se quiere hacer énfasis es que más de la mitad de la producción de artículos científicos está relacionada con instituciones universitarias, y se deja de lado la producción que se reconoce o que se debe generar por parte de sociedades científicas, como lo es la Sociedad Colombiana de Cardiología y Cirugía Cardiovascular, perspectiva que no implica que los miembros de las sociedades científicas no estén generando producción de documentos científicos, sino que éstas se desarrollan como parte de grupos de investigación adscritos a las universidades en donde se tiene actividad docente, y es por este motivo que no se publica en representación o a nombre de alguna de las sociedades a la cual pueda pertenecer.

Jara-Navarro ${ }^{1}$, sin embargo, permite entrever una necesidad que va mucho más allá de lo relacionado con la investigación para la salud, debido a que plantea que no basta con investigar y publicar los resultados de dichas investigaciones, sino que además es necesario invertir esfuerzos y recursos en el uso óptimo y la apropiación de esos resultados. Con base en este planteamiento, cobra relevancia visionar la investigación no solo como un medio para dar a conocer la producción científica, sino como área de conocimiento en sí misma.

Al considerar la necesidad de investigar e innovar en salud, se debe evaluar la oportunidad inmensa que se tiene de construir equipos interdisciplinarios e interinstitucionales ${ }^{5}$, para obtener resultados en investigación e innovación como fuente de riqueza social y económica.

Así mismo, se plantea la pertinencia de la formación a los profesionales de la salud en áreas de investigación ${ }^{5}$, innovación y tecnología, en pregrado, posgrado y actividades de formación continuada, además del fortalecimiento de las competencias en un segundo idioma, preferiblemente inglés, lectura crítica de literatura científica, y en competencias en aplicación y apropiación correcta de la información.

En concordancia con lo anterior, en 2008 se llevó a cabo la "Primera conferencia latinoamericana sobre investigación e innovación para la salud" ", en la cual se establecieron algunos lineamientos básicos, entre los cuales cabe resaltar los siguientes:

- Articulación de la investigación y el desarrollo a través del trabajo conjunto de diferentes actores tanto estatales como civiles, así como trabajo en la formación y capacitación del talento humano en estos temas, con énfasis en un enfoque de formación - investigación - acción y no solo con un enfoque teórico a fin de mantener este tipo de iniciativas.

- Formación de equipos interdisciplinarios y no únicamente de equipos con fortalezas técnicas y académicas en una sola disciplina como la Medicina; al igual que búsqueda de cooperación internacional y articulación de la investigación con los sectores productivos y el trabajo a través de redes. 
- Los retos de la salud pueden transformarse en oportunidades mediante innovaciones tecnológicas y sociales que no son necesariamente costosas.

- Cuestionamiento a la publicación como forma tradicional para evaluar los resultados de producción investigativa e innovadora.

- Planteamiento de la idea de la innovación a través del descubrimiento mediante investigación básica, desarrollo de herramientas nuevas y mejoradas en aspectos preclínicos y clínicos, y distribución de los productos y resultados a los pacientes.

En 2011, tuvo lugar la "Segunda conferencia latinoamericana sobre investigación e innovación para la salud," " 7 en la cual se complementaron algunos de los planteamientos hechos en la primera versión, que se destacan a continuación:

- La innovación no necesariamente implica alta inversión, sino soluciones rentables y de alto impacto a las problemáticas en salud.

- Se separan los conceptos de investigación en salud e investigación para la salud, el primero relacionado con la investigación biomédica y el segundo con la aplicación de cualquier otra área a la salud.

- Existe necesidad de articular la investigación con políticas en salud.

- Es esencial fomentar la formación de investigadores jóvenes en investigación y desarrollo.

- La importancia de la multidisciplinariedad y la multisectorialidad son fundamentales para el desarrollo exitoso tanto de la investigación como de la innovación.

- La innovación debe ser concebida no solo como el desarrollo de nuevos productos, sino también de nuevos servicios o procesos.

Lo anterior permite evidenciar cómo en Latinoamérica se realizan actividades encaminadas a fortalecer la investigación y la innovación, pero no son difundidas de manera adecuada y amplia, lo cual impide que cualquier propuesta hecha en este tipo de escenarios se ejecute y se aplique en la práctica diaria de los trabajadores del área de la Medicina.

Otra forma de ver el problema relacionado con el poco conocimiento que existe de esfuerzos internacionales concernientes a la investigación e innovación en el área de la salud, radica en el poco interés que se muestra por conocer y participar de estas iniciativas, debido a que se suele creer que la investigación y la innovación en salud son responsabilidad de actores como universidades, grupos de investigación, entidades, epidemiólogos, industria u otros.

Por otro lado, llaman la atención la forma cómo en los últimos años viene hablándose de investigación en asociación con innovación, y aunque en el área médica se aprecian resultados, es claro que el trabajo en innovación marca una de las metas a las cuales se debe comenzar a apuntar.

Frente al tema de innovación se presenta el concepto propuesto por el Manual de Oslo ${ }^{8}$ que la considera como la introducción de un nuevo, o significativamente mejorado, producto (bien o servicio), de un proceso, de un método de comercialización o de un nuevo método organizativo, en las prácticas internas de la empresa, la organización del lugar de trabajo o las relaciones exteriores.

En $2018^{9}$ se presenta el undécimo informe del Índice de Innovación Mundial, que si bien en esta última versión se enfoca en el desarrollo de avances en lo relacionado con el sector energético, muestra una excelente evaluación de la innovación mundial a través de diferentes indicadores que permiten calcular el desarrollo de este tema de acuerdo con las diferentes regiones del mundo.

El mundo se divide en siete regiones; en el quinto lugar se encuentran América Latina y el Caribe, los tres primeros puestos están ocupados por Chile, Costa Rica y México. Colombia ocupa el puesto 63 de 126 países con una puntuación de 33,78 de 100 puntos posibles a evaluar.

En este documento, se plantea el trabajo en innovación a través de cinco ejes:

- Institucionalidad, desde lo gubernamental, lo regulatorio y lo empresarial.

- Capital humano e investigación, con énfasis en educación, investigación y desarrollo.

- Infraestructura.

- Sofisticación del mercado, con referencia a la posibilidad económica a través de créditos, inversión y competencia económica.

- Sofisticación empresarial, en cuanto a trabajadores del conocimiento, alianzas para la innovación y absorción del conocimiento.

Se plantean dos categorías adicionales en lo que respecta a los resultados. La primera se relaciona con conocimiento y tecnología en la cual se desagregan aspectos de creación, impacto y difusión; la segunda tiene que ver con los resultados en creatividad enfocados en activos intangibles, bienes y servicios creativos, y creatividad a través de la virtualidad.

Acerca de estas necesidades para la innovación se hace una medición por regiones del mundo, en la cual se identifican ocho. América Latina y el Caribe ocupan el quinto lugar en institucionalidad, el séptimo en capital humano e investigación, el sexto en infraestructura y en sofisticación del mercado, el quinto en sofisticación empresarial, el séptimo en resultados relacionados a conocimiento y tecnología, y el sexto en resultados en creatividad.

En el contexto actual la innovación se percibe como un punto determinante del desarrollo económico, la competitividad y la transformación de las sociedades ${ }^{10}$, y pese a que sin lugar a dudas la investigación está ligada a los sistemas políticos debido a que la salud es en sí misma un derecho y un objetivo del desarrollo sostenible, es precisamente este dilema el que ha invitado a otras áreas disciplinares a intervenir en los procesos de investigación e innovación para la salud.

Como se mencionó, desarrollar el tema de innovación sin contextualizarlo a la realidad nacional carece de un propósito. Por este motivo se considera importante presentar uno de los ejes operativos del Modelo Integral de Atención en Salud (MIAS) ${ }^{11}$, que es precisamente el fortalecimiento de la investigación, innovación y apropiación de conocimiento, bajo principios rectores de interdisciplinariedad, pertinencia y efectividad. Dentro del modelo se proponen además seis retos desde la perspectiva de innovación para la salud: 
- Establecimiento de la rectoría del sistema de salud.

- Estrategias que garanticen la sostenibilidad financiera del sistema de salud.

- Gestión eficiente de los recursos humanos en salud.

- Fortalecimiento de los sistemas de información efectivos y eficientes.

- Mecanismos efectivos de transferencia de conocimiento en políticas públicas.

- Investigación en acceso, utilización y garantía de la calidad de la prestación de servicios de salud.

De esta manera, se muestra nuevamente cómo en los planos institucional y Estatal han venido planteándose bases de trabajo para los temas de innovación e investigación, y cómo dichas bases no son gestionadas de manera adecuada por los profesionales del área de la salud y menos aún por los profesionales y especialistas de la Medicina, evidenciándose en el hecho que mucho se ha socializado y abordado del MIAS ${ }^{11}$, pero este eje operativo ha sido de los menos divulgados y conocidos.

Una vez que se han discutido ampliamente los temas de investigación e innovación en el contexto de la salud, con énfasis en las necesidades que existen en este sentido, se comparte la posición de $Z_{\text {árate }}{ }^{5}$, en la cual se plantea que los procesos y los objetos de investigación en salud son dinámicos y multifactoriales, lo que hace que sean idóneos para el abordaje desde una perspectiva de innovación y permiten definir la gran oportunidad que tienen todos los profesionales y especialistas de la Medicina frente a dichos tópicos.

Esta oportunidad debe aprovecharse al máximo, lo cual depende directamente de la existencia de profesionales formados y bien capacitados, motivados y con posibilidad de desarrollar proyectos de investigación e innovación alineados con las necesidades del país, enfocados siempre en el crecimiento continuo de la salud y la Medicina, de cara a la responsabilidad que tiene cada profesional en su actividad laboral y académica diaria en este contexto.

Es así como este editorial es una invitación para seguir trabajando en procesos y diferentes sectores de investigación e innovación de alta calidad, no planteada como un ideal de la práctica sino como una realidad y una necesidad del crecimiento profesional, yendo más allá de investigaciones "de papel" con la finalidad de esperar una publicación, sino con una motivación basada en el deseo de construir conocimiento, incitar el pensamiento crítico y estimular el mejor aprovechamiento de las oportunidades.

Para finalizar, un buen ejemplo de los temas de investigación e innovación se presenta en el contexto de la investigación clínica ${ }^{12}$, que aún después de tantos años de ejecución en el país, existen estigmas y desaprobación por muchas personas, incluso profesionales de la salud y de la Medicina, desconociendo que ésta representa para el país un motor de proyección económica, académica y social, y que permite acceder y desarrollar competencias investigativas e innovadoras.

Es así como en lo estatal y privado, diferentes entidades como MINSALUD, INVIMA, Comercio Exterior, Asociación Colombiana de Centros de Investigación Clínica (ACIC), el Instituto de Evaluación Tecnológica en Salud (IETS), AVANZAR y AFIDRO realizan constantes esfuerzos para el fortalecimiento de la investigación clínica, sin embargo, ante esta lucha, la responsabilidad es precisamente aprovechar al máximo estas oportunidades y lograr así posicionar al país como un modelo de investigación e innovación para la salud.

\section{Bibliografía}

1. Jara-Navarro MI. El reto de la investigación y la producción científica en salud. Revista Gerencia, Política y Salud. 2012;11:5-10.

2. Scimago Journal \& Country Rank [Internet]. Scimago Journal \& Country Rank [Consultado en: 23 de marzo de 2019]. Disponible en: https://www.scimagojr.com/.

3. COLCIENCIAS [Internet]. Publindex. Indexación - homologación. [Consultado en: 23 de marzo de 2019]. Disponible en: https://scienti.colciencias.gov.co/publindex/.

4. Maz-Machado A, Jiménez-Fanjul N, Villarraga M. La producción científica colombiana en SciELO: un análisis bibliométrico. Revista Interamericana de Bibliotecología. 2016;39:111-9.

5. Zárate E. Innovación en la investigación. Rev Peru Med Exp Salud Publica. 2010;27:432-7.

6. Comité Ejecutivo, Primera Conferencia Latinoamericana sobre Investigación e Innovación en Salud 2008. $1^{a}$ conferencia Latinoamericana sobre Investigación e Innovación para la salud. Informe de la conferencia, resultados y documentos. 2008.

7. Consejo de Investigación en Salud para el Desarrollo (COHRED). 2 a Conferencia Latinoamericana sobre Investigación e Innovación para la Salud. 2011.

8. OCDE, Eurostat. Manual de Oslo. Guía para la recogida e interpretación de datos sobre innovación. Tercera Edición. Editorial Grupo Tragsa (Madrid - España). 2006.

9. Cornell University, INSEAD, WIPO (World Intellectual Property Organization): The Global Innovation Index 2018: Energizing the World with Innovation. Fontainebleau, and Geneva: Ithaca; 2018.

10. Silveira-Costa L, Salud. desarrollo e innovación. Cad. Saúde Pública. 2016;32(Sup 2), eED01S216.

11. Ministerio de Salud y Protección Social. Política de Atención Integral en Salud. Bogotá D.C., 2016.

12. Molina de Salazar DI, Álvarez-Mejía M. Estado de la investigación clínica en Colombia. Acta Med Colomb. 2018;43:179-82. 